\title{
Determination of the Energy of a Primary Particle in Accordance with the Hypothesis of Primary Particles and Another Meaning of Planck Mass
}

\author{
Slobodan Spremo \\ Mathematical Grammar School, Belgrade, Serbia \\ Email: slobodan.spremo@gmail.com
}

How to cite this paper: Spremo, S. (2021) Determination of the Energy of a Primary Particle in Accordance with the Hypothesis of Primary Particles and Another Meaning of Planck Mass. Journal of High Energy Physics, Gravitation and Cosmology, 7, 144-148.

https://doi.org/10.4236/jhepgc.2021.71007

Received: October 11, 2020

Accepted: January 10, 2021

Published: January 13, 2021

Copyright $\odot 2021$ by author(s) and Scientific Research Publishing Inc. This work is licensed under the Creative Commons Attribution International License (CC BY 4.0).

http://creativecommons.org/licenses/by/4.0/

\section{(c) (i) Open Access}

\begin{abstract}
This paper represents a continuation of our Hypothesis of primary particles, which provides an opportunity for describing the origin of the Big Bang and other universes. In its hypothesis, we have shown that there was a possibility of hypothetical primary particles moving in their own flat spacetime, in their basic, dynamic state and possessing speeds much higher than the speed of light, acquiring energy and momentum during deceleration in mutual collisions, which would tunnel into various universes. The cosmic microwave background is evidence that our universe expanded from a very hot, dense state, which is consistent with our hypothesis. The lower border speed to which a primary particle at the Big Bang came very close in a collision during its deceleration, simultaneously represents the upper border speed in our Universe-the speed of light in a vacuum. The speed of light, along with other fundamental physical constants, had shaped our Universe, in a manner in which we are still able to recognize the "physical gene" that preceded our existence. By virtue of comprehending our Universe, using the help of fundamental physical constants, we have determined that the mass attributed to the primary particle, in accordance with the Hypothesis of primary particles, would correspond to the Planck mass. Therefore, energy of the primary particle would be: $E_{p} \approx 1.22 \times 10^{19} \mathrm{GeV}$.
\end{abstract}

\section{Keywords}

Big Bang, Flat Spacetime, Planck Mass, Quantum of Speed

\section{Introduction}

According to the Hypothesis of primary particles [1], they exist in their own flat spacetime, in their basic, dynamic state, i.e. while moving at speeds $u_{p} \gg c$, 
possessing energy

$$
E_{p}=m_{p} c^{2}
$$

where $m_{p}$ represents the attributed mass of the primary particle. Further, when these particles slow down to lesser speeds $u$, their overall energy $E_{t}$ and momentum $p_{p}$ increase in accordance with the following relations:

$$
E_{t}=\frac{m_{p} c^{2}}{\sqrt{1-\frac{c^{2}}{u^{2}}}}=\xi m_{p} c^{2},
$$

and

$$
p_{p}=\frac{m_{p} c^{2}}{u \sqrt{1-\frac{c^{2}}{u^{2}}}}=\frac{\xi m_{p} c^{2}}{u},
$$

so that:

$$
p_{p}=\frac{E_{t}}{u} .
$$

Thus, the possibility exists that about 13.65 billion years ago, during the deceleration of some of these particles caused by their mutual collisions to a speed slightly higher than the speed of light, enormous energy and momentum it possessed, as observed in the above relations, were tunnelled through the Big Bang into our Universe, creating our own energy and mass. We consider the speed of light in a vacuum $c$ to be the border speed, so the photons in our Universe, by the Special Theory of Relativity, cannot achieve this border speed [2], but a speed slightly lower than it instead, i.e. the speed that is by the "quantum of speed" $\varepsilon_{v} \approx 2.38 \times 10^{-114} \mathrm{~m} \cdot \mathrm{s}^{-1}$ lower than the border speed $c$. In addition to the "quantum of speed", in this paper, we will also utilise the data on the mass of our Universe $m_{U} \approx 1.73 \times 10^{53} \mathrm{~kg}$. This mass includes every type of mass (baryonic and dark mass) and the mass associated with all types of energy (photons, dark energy, etc.).It is important to note that this would be the mass of the Universe from the perspective of the observer in our position in the Universe, which would differ for the observers depending on their relative movement in relation to us, so e.g. for an observer travelling with a photon that would represent the Planck mass. For the Planck mass, we will use $m_{P}=\sqrt{\frac{\hbar c}{G}} \approx 1.22 \times 10^{19} \mathrm{GeV} / c^{2} \approx 2.18 \times 10^{-8} \mathrm{~kg}$.

As we can see, the creation of other universes could occur with a certain primary particle slowing down very close to some other border speed $k$, which would determine these universes in accordance with different physical constants, and therefore different properties and incomprehensible to us.

2. The Energy Possessed by the Primary Particle in Its Basic State While Moving at the Speed $u_{p}$

\subsection{Derivation of the Value of the mass Attributed to the Primary}




\section{Particle from Its Energy}

Due to the increase in the energy of the primary particles during their deceleration, it can be stated that, in contrast to the kinetic energy, they increase their homeokinetic energy $H$ with their speed decreasing $H=E_{t}-E_{p}$. Hence, close to the border speed $c, H \rightarrow E_{t}$. At this moment, the total energy of the primary particle $E_{t}$ is tunnelled into the total energy of our created Universe $E_{U}$, i.e.

$$
E_{t}=E_{U} .
$$

Considering that according to [2]:

$$
m_{P}=m_{U} \sqrt{1-\frac{\left(c-\varepsilon_{v}\right)^{2}}{c^{2}}},
$$

and similar derived from (2) and (5), we can note that:

$$
\frac{m_{p} c^{2}}{\sqrt{1-\frac{c^{2}}{u^{2}}}}=m_{U} c^{2}
$$

during which $u \rightarrow c$, i.e.

$$
m_{p}=m_{U} \sqrt{1-\frac{c^{2}}{\left(c+\varepsilon_{u}\right)^{2}}}
$$

where $\varepsilon_{u}$ represents the "speed quantum of the primary particle".

Further, from (6) and (8) we see that:

$$
\frac{m_{p}}{\sqrt{1-\frac{c^{2}}{\left(c+\varepsilon_{u}\right)^{2}}}}=\frac{m_{P}}{\sqrt{1-\frac{\left(c-\varepsilon_{v}\right)^{2}}{c^{2}}}}=m_{U}
$$

and logically assuming that $\left(\varepsilon_{u} \ll c\right)$, we can conclude that the attributed mass of the primary particle is equal to the Planck mass

$$
m_{p}=m_{P} .
$$

Similarly, using (9) we can determine "speed quantum of the primary particle" from

$$
\frac{c}{c+\varepsilon_{u}}=\frac{c-\varepsilon_{v}}{c},
$$

as

$$
\varepsilon_{u}=\frac{c \varepsilon_{v}}{c+\varepsilon_{v}} \approx 2.38 \times 10^{-114} \mathrm{~m} \cdot \mathrm{s}^{-1} .
$$

\subsection{Deriving the Value of the Mass Attributed to the Primary Particle from Its Momentum}

From (3), we can now express the mass of the primary particle as:

$$
m_{p}=\frac{p_{p} u}{c^{2}} \sqrt{1-\frac{c^{2}}{u^{2}}} .
$$


In accordance with the law of momentum conservation, the primary particle transmitted its entire momentum to our Universe during the Big Bang, i.e.

$$
p_{p}=p_{U},
$$

where, according to [2] $p_{U}=m_{U} c$, we have:

$$
m_{p}=\frac{m_{U} c\left(c+\varepsilon_{u}\right)}{c^{2}} \sqrt{1-\frac{c^{2}}{\left(c+\varepsilon_{u}\right)^{2}}}=m_{P} .
$$

Thus, this way we also concluded that the attributed mass of the primary particle is equal to the Planck mass.

Therefore, the primary particle energy according to (1) equals:

$$
E_{p} \approx 1.22 \times 10^{19} \mathrm{GeV} \text {. }
$$

With the primary particle energy determined in this manner, our hypothesis gains a more complete meaning.

\section{Result}

In this paper, we have determined the attributed mass of the primary particle that is the Planck mass, which has now acquired this new meaning. This mass was determined in two ways, by utilising the expressions for energy and for the momentum of the primary particle. Certainly, the energy of these particles in their basic state, i.e. while moving at their maximum speeds $u_{p}$, is now known. We based our considerations on the Hypothesis of primary particles and [2], which, in addition to the well-known ones, also provided new connections between fundamental physical constants.

The cosmic microwave background is evidence that our Universe has expanded from a very hot, dense state, which is in line with our hypothesis.

\section{The Need for Development of a Primary Particle Hypothesis and Their Experimental Proof}

In addition to the previous dynamic properties of these particles, we have now determined their energy, as well. Therefore, we believe that further study of hypothetical primary particles could make an important scientific shift in physics, as well as in our philosophical view of the world.

Given the described properties of primary particles, we expect their indirect experimental evidence to be possible through a successful explanation of the Big Bang, via further scientific work on the Hypothesis of primary particles.

\section{Conflicts of Interest}

The author declares no conflicts of interest regarding the publication of this paper.

\section{References}

[1] Spremo, S. (2019) Hypothesis of Primary Particles and the Creation of the Big Bang 
and Other Universes. Journal of Modern Physics, 10, 1532-1547.

https://doi.org/10.4236/jmp.2019.1013102

[2] Mercier, C. (2019) Calculation of the Mass of the Universe, the Radius of the Universe, the Age of the Universe and the Quantum of Speed. Journal of Modern Physics, 10, 980-1001. https://doi.org/10.4236/jmp.2019.108065 